\title{
Shift-free fixed-line laser protection filter technology
}

Wang, James

DOI:

$10.1117 / 12.2573993$

Published: 07/10/2020

Early version, also known as pre-print

Cyswllt i'r cyhoeddiad / Link to publication

Dyfyniad o'r fersiwn a gyhoeddwyd / Citation for published version (APA):

Wang, J. (2020). Shift-free fixed-line laser protection filter technology. Paper presented at

Technologies for Optical Countermeasures XVII; and High-Power Lasers: Technology and

Systems, Platforms, Effects IV. https://doi.org/10.1117/12.2573993

\footnotetext{
Hawliau Cyffredinol / General rights

Copyright and moral rights for the publications made accessible in the public portal are retained by the authors and/or other copyright owners and it is a condition of accessing publications that users recognise and abide by the legal requirements associated with these rights.

- Users may download and print one copy of any publication from the public portal for the purpose of private study or research.

- You may not further distribute the material or use it for any profit-making activity or commercial gain

- You may freely distribute the URL identifying the publication in the public portal ?
}

Take down policy

If you believe that this document breaches copyright please contact us providing details, and we will remove access to the work immediately and investigate your claim. 


\title{
Shift-free fixed-line laser protection filter technology
}

\author{
James N. Monks*ab , Jonathan Williams ${ }^{\mathrm{a}}$, Andrew Hurst ${ }^{\mathrm{a}}$, Zengbo Wang ${ }^{\mathrm{b}}$ \\ ${ }^{a}$ Qioptiq Ltd., St. Asaph, Denbighshire, United Kingdom. LL17 0LL; ${ }^{b}$ School of Electronic \\ Engineering, Bangor University, United Kingdom. LL51 1UT.
}

\begin{abstract}
The development of shift-free fixed-line filters is a key technology for advancing the next generation of laser protection and is greatly desired due to the increased threat of laser attacks. Thin-film interference coatings have remained the key technology for achieving narrow bandstop filters for protection against laser light since the late 1970s. This paper presents the latest developments in fixed-line laser technology and introduces a metamaterial solution to mitigate the angular shift found in thin-film interference coatings. The metamaterial coating consists of metallic nano-particles periodically distributed within a non-absorbing dielectric material with a specific refractive index that enables the desired plasmonic resonance to exist at wavelengths that match that of the lasers. Due to the nano-particle size, the metamaterial layer can be treated as an individual homogeneous layer with properties described by an effective Drude-Lorentz approximation model. Unlike standard interference coatings where the effective index of the stack decreases with larger angles of incidence, the metamaterial's effective index remains relatively fixed with increasing angles resulting in the narrow bandstop function remaining shift-free.
\end{abstract}

Keywords: Laser dazzling countermeasures, Laser protection filters, Novel optical filters, Wide-angle protection, Metamaterials, Effective medium approximations.

\section{INTRODUCTION}

Fixed-line laser protection filters are widely used in many applications including defence, medical, manufacturing and research fields [1]. They are narrow bandstop filters, often referred to as notch filters, which are specifically designed to protect against laser wavelengths and often have a small bandwidth to allow for the highest possible integrated visual transmission [2]. Notch filters can achieve high reflections within the rejection band and offer high transmission outside the rejection band. Laser protection filters can be used to shield sensors as well as the human eye, and can provide safety against wavelengths in the UV-Vis-IR spectrums. The notch filter is a combination of a longwave pass and a shortwave pass filter. The general design follows alternating layers of high- and low-index materials, each at a thickness of a quarter wavelength to create partially reflected waves at each interface. The reflections recombine through constructive interference, and fashion a high-quality reflector.

Although the existing thin-film technology has been the staple for laser protection systems and presents considerable advantages, they also have some fundamental limitations. The main deficiency with thin-films is the angular sensitivity $[2,3]$. As the angle of incidence increases, the transmission spectrum of the filter experiences a continuous shifting to shorter wavelengths. This results in the failure of the device to operate at the desired blocking wavelength. To circumvent this issue, the thin-film filters are designed with an extended bandwidth to allow coverage over the desired blocking wavelength as the shifting occurs. This in turn creates further problems through reduced visibility (decrease in transmission) and high coloration. Therefore, it has become highly desirable to find a solution that is capable of combating the limits found in standard thin-film technology [4].

In recent years, metamaterials have been highlighted as materials of the future, and are defined as man-made media designed with specific functions. Ordinary materials, i.e. material that exist in nature, respond to an electromagnetic field in accordance to how the atoms and molecules polarise, with their average behavior over the lattice providing the electromagnetic response. In contrast, metamaterials replace the atoms and molecules with larger elements that display a physical structure. These units are known as meta-atoms and remain much smaller than the wavelength of interest. By existing at a much smaller size, compared to the wavelength, they begin to act like atoms and molecules. Thus, by designing the shape, geometry, size, orientation and arrangement, it becomes possible to envisage the properties and responses of the metamaterials. The major advantage is the fact that they are not limited to the chemistry of the periodic table and allow for another dimension of design [5-7]. 
This paper details the limitations of thin-film interference filters for laser protection applications and introduces a metamaterial approach for enabling shift-free fixed-line technology. Due to the complexities of designing metamaterials, this research highlights an effective medium approximation (EMA) for dispersive metamaterials that display metallic inclusions through an effective Drude-Lorentz model. The Drude-Lorentz model was adapted for an effective medium design as current favorable models, such as Maxwell-Garnett theory [8,9], are inadequate for describing dispersive properties. The EMA model enables the metamaterial to exist in a one-dimension narrative that can be used with wellestablished thin-film design tools including the transfer matrix method. The metamaterial has been designed with a resonant frequency that matches the laser wavelength, with analysis at oblique angles demonstrating a fixed effective refractive index modulus, permitting a fixed central wavelength across all incidence angles. Additional dielectric thinfilms have been added to the metamaterial to improve the overall transmission properties outside the bandstop region. As such, this research demonstrates the capability of using metamaterials with dielectric thin-films to execute a high-quality optical filter for applications in laser protection.

\section{ANGLE-SENSITIVE THIN-FILM FILTERS}

The disparity of performance of an all-dielectric thin-film filter with incidence angles is a well-known effect and has been widely studied $[2,10]$. The central wavelength shift is inversely proportional to the effective refractive index of the thin-film filter.

$$
\lambda_{\theta}=\lambda_{0}\left(1-\frac{\sin ^{2}(\theta)}{n_{e f f}^{2}}\right)
$$

Where $\lambda_{0}$ (respectively $\lambda_{\theta}$ ) is the central wavelength of the filter at normal incidence (respectively oblique incidence), $\theta$ the incidence light in air and $n_{\text {eff }}$ the effective refractive index of the filter.

The effective refractive index of the narrowband thin-film filter design can be described by (Eq.2), with dependence on the nature of the film spacer construction. The effective index varies with $m$, the interference order of the filter and $n_{L}$ and $n_{H}$, the refractive indices of the quarter-wave alternated layers.

$$
n_{\text {eff }}= \begin{cases}n_{H} \sqrt{\frac{m-(m-1)\left(n_{L} / n_{H}\right)}{(m-1)-(m-1)\left(n_{L} / n_{H}\right)+\left(n_{H} / n_{L}\right)}} & \text { for high-index spacers } \\ n_{L} \sqrt{\frac{m-(m-1)\left(n_{L} / n_{H}\right)}{\left(m-m\left(n_{L} / n_{H}\right)\right)+\left(n_{L} / n_{H}\right)^{2}}} & \text { for low-index spacers }\end{cases}
$$

The thin-film layers within the filter remain at a fixed thickness. However, as the angle of incidence increases, the path of the light rays increases through the film layers. Consequently, this change in ray path length with respect to oblique incidence angles can be described as a change in effective index of the filter and can be simply described by (Eq.3).

$$
n_{e f f}(\theta)=\sqrt{n_{e f f}^{2}-\sin ^{2}(\theta)}
$$

In an ideal situation, if the effective index can remain fixed with varying angles of incidence, then the filter would not experience a central wavelength shift. Often to help moderate the shifting behavior, the effective index should be as high as practically possible.

A design has been constructed to demonstrate the effects of angular performance of a typical thin-film laser protection filter, and the configuration can be found in (Eq.4). The formation stack consists of 56 layers that alter from low $(L)$ to high $(H)$ index materials with a reference wavelength of $532 \mathrm{~nm}$. The indices of refraction are 1.48 and 1.772 , with an effective index of 1.607 .

$$
\text { Air }\left|L(H L)^{27} L\right| \text { Glass }
$$

The typical curves are displayed in figure 1 and show how the central wavelength and effective refractive index of a thinfilm filter, centered at $532 \mathrm{~nm}$, varies with the angle of incidence. 

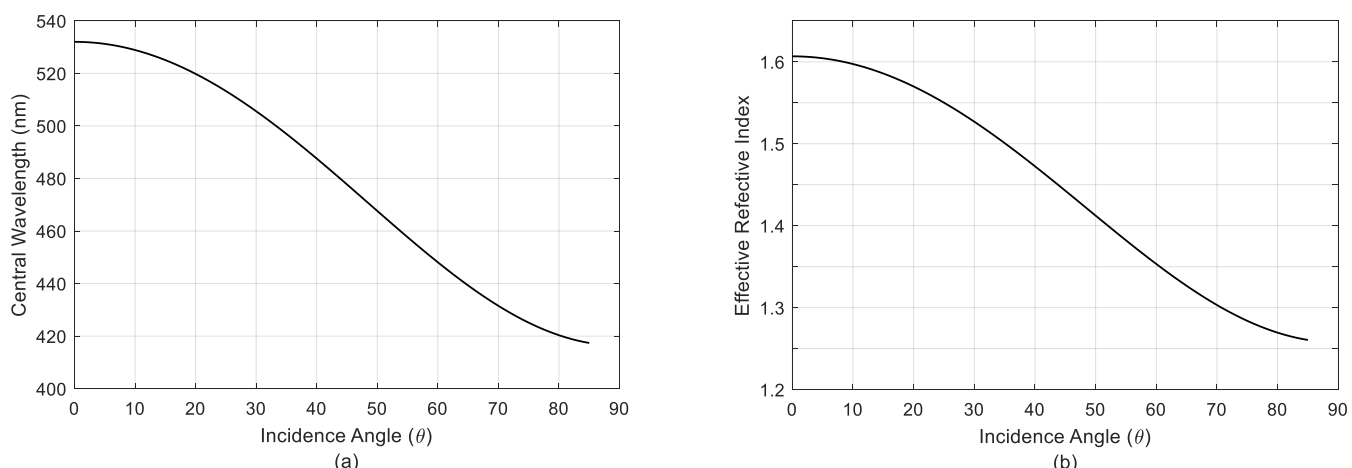

Figure 1. (a) Shift of the peak wavelength with angle of incidence. (b) Shift in the stacks effective refractive index with angle of incidence.

Although the polarisation shifts at the same rate, they do present a difference when considering attenuation. As the notch feature shifts with angle, the S- and P-polarisation states began to separate, with a slightly narrowing of the filters function for the P- state and a similar broadening for the S- state. Figure 2 shows the features of such effects for the filter described by (Eq.4). Additionally, Figure $2 \mathrm{~b}$ also show how the filters function no longer provides protection with increased incidence angles at the desired $532 \mathrm{~nm}$ laser wavelength.

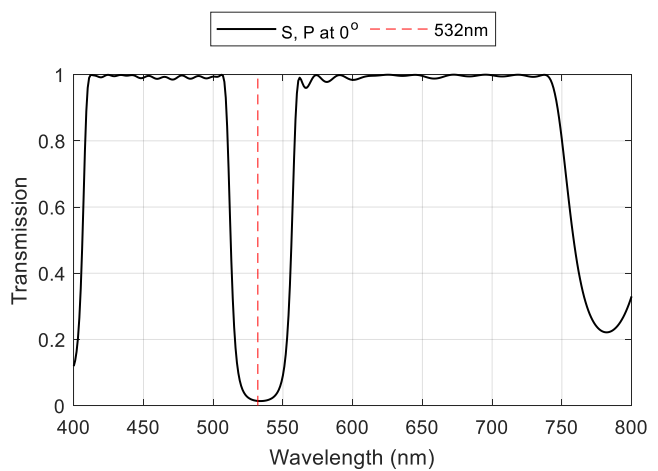

(a)

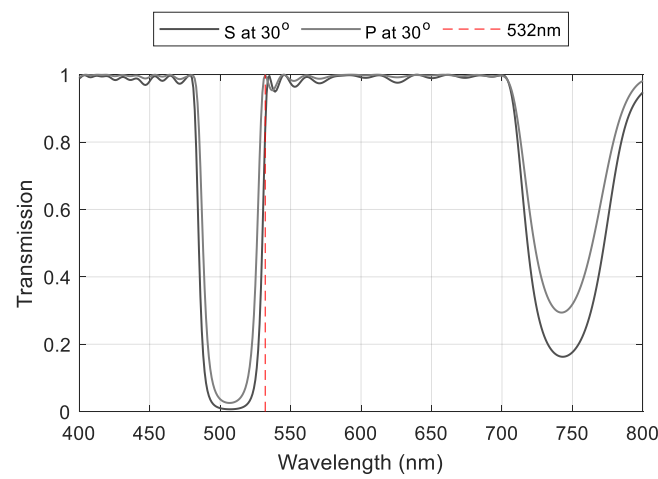

(b)

Figure 2. The transmission response with consideration to the polarisation effects on standard thin-film filters.

Figure 3 highlights the mean transmission shift function of the filter at incidence angles 0, 25 and 50 degrees. The dotted line represents the laser wavelength. The figure focuses on the bandstop region to demonstrate how the notch location is unable to provide adequate protection against the $532 \mathrm{~nm}$ laser wavelength. At normal angles of incidence, the central wavelength is located at $532 \mathrm{~nm}$; as the angle increases, the narrow notch shifts towards the blue wavelengths leaving the $532 \mathrm{~nm}$ wavelength exposed for transmission through the filter. 


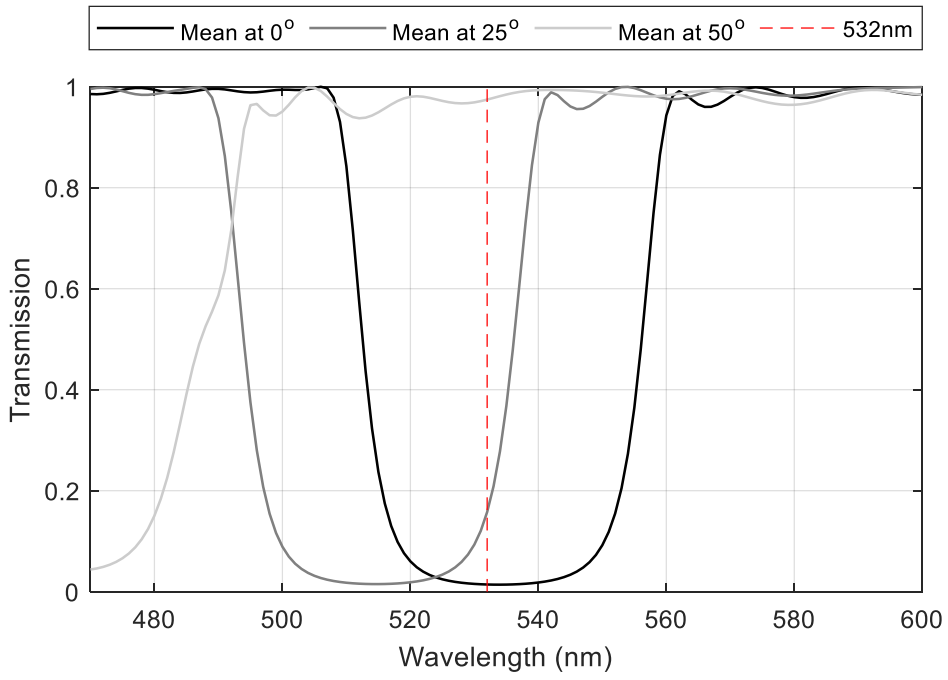

Figure 3. The mean transmission function of a standard thin-film filter, focusing on the bandstop region.

The blocking performance of a filter is often measured from the optical density (OD) of the filter and is a function of the transmission $(\mathrm{T})$.

$$
O D=-\log _{10}(T)
$$

The optical density terms the degree to which a medium impedes transmitted light waves and is commonly used within the optical industry to define laser safety guidelines. Figure 4 shows the calculated optical density of the filter for angles between 0 and 85 degrees at a fixed wavelength of $532 \mathrm{~nm}$. This representation also demonstrates the shifting behavior of a filter. As such, the filter discussed is capable of providing +1.5OD to 20 degrees. At angle ranges of 20 to 30 degrees the notch passes over the $532 \mathrm{~nm}$ wavelength. Once at 30+ degrees, the notch's central wavelength is $\sim 505 \mathrm{~nm}$ and proceeds to $\sim 420 \mathrm{~nm}$ as the angle increase. As such, the filter no longer provides any protection against the laser wavelength.

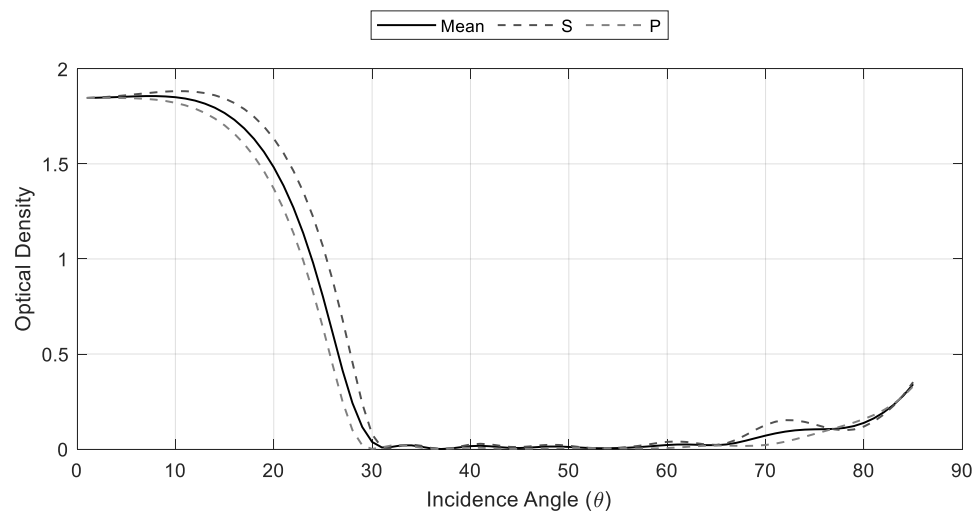

Figure 4. Optical density as a function of incidence angle at a fixed wavelength of $532 \mathrm{~nm}$.

\section{EFFECTIVE MEDIUM APPROXIMATIONS FOR METAMATERIAL THIN-FILMS}

Dispersive and resonant effects are principle properties in plasmonic metamaterial applications. A key principle is the ability to tailor the dispersive response. When a plasmonic particle is surrounded by a dielectric medium, the plasmonic resonance redshifts. The strength of the shift depends on the refractive index of the surrounding material [11]. Another influencing feature relates to the fill fraction (Eq.6) which is governed by the dimension of the meta-atom. However, the increase in fill fraction percentage results in high permittivity solutions. The subwavelength particles with high positive dielectric permittivity support strong resonances with a large contra-directional displacement current that can induce 
unwanted strong magnetic fields $[12,13]$. Therefore, it is preferable to tailor the resonant wavelength by varying the surrounding refractive index.

$$
p=\frac{\text { Volume of solid part of film }}{\text { Total volume of film }}
$$

Effective medium approximations are becoming a major consideration when designing metamaterials [14] because they allow complex metamaterial descriptions to be described by a single uniformed layer. This process is known as homogenisation. The Maxwell-Garnett theory (MGT) is commonly used to describe composite metamaterials [15] and their macroscopic properties for a uniformed composite with two mixed elements. Typically for electromagnetic properties, the MGT describes the bulk effective permittivity of a metamaterial in terms of the inclusion materials and the host materials. However, the MGT displays limitations, particularly when considering dispersive materials. Periodic elements with dispersive properties can traditionally be described with several constants that can be formulated into either a Drude equation or a Drude-Lorentz equation; depending whether the material is metallic, semiconductor or dielectric.

Unlike the MGT formula that requires the entire dispersive permittivity function for each present material, with respect to wavelength, in order to calculate an effective dispersive permittivity; the effective Drude-Lorentz formula enables the input constants described by a typical Drude style material and formulates new effective constants to described the dispersive metamaterial.

$$
\varepsilon_{e f f}(\omega)=\varepsilon_{\infty, e f f}+\frac{\omega_{p, e f f}^{2}}{\omega_{0, e f f}^{2}-\omega^{2}+i \omega \Gamma}
$$

Where the effective constant offset (high frequency) permittivity $\varepsilon_{\infty, e f f}$, plasma frequency $\omega_{p, e f f}$, and the resonance frequency $\omega_{0, e f f}$, formulas are described below.

$$
\begin{gathered}
\varepsilon_{\infty, e f f}=\varepsilon_{h}+3 p \varepsilon_{h}\left(\frac{\varepsilon_{\infty}-\varepsilon_{h}}{\varepsilon_{\infty}+\chi \varepsilon_{h}-p\left(\varepsilon_{\infty}-\varepsilon_{h}\right)}\right) \\
\omega_{p, e f f}=\sqrt{p}\left(\frac{3 \varepsilon_{h}}{(1-p) \varepsilon_{\infty}+(\chi+p) \varepsilon_{h}}\right) \omega_{p} \\
\omega_{0, e f f}=\left(\sqrt{\frac{1-p}{(1-p) \varepsilon_{\infty}+(\chi+p) \varepsilon_{h}}}\right) \omega_{p}
\end{gathered}
$$

With $\omega$ indicating angular frequency, $\varepsilon_{h}$ denoting the permittivity of the host medium, $p$ describing the fill fraction and $\chi$ describing the polarisability of the particle which is determined by the particle shape. The remaining parameters $\left(\varepsilon_{\infty}, \omega_{p}\right.$ and $\Gamma)$ are defined from the Drude type particle.

The morphology of the particle can influence the plasmonic behavior, either by shifting the response or creating multiple resonance positions that can be electromagnetically polarisation sensitive. For spherical particles, $\chi=2$.

The conversion from permittivity to refractive index can be calculated from (Eq.11), where $n$ equals the effective refractive index and $k$ equals the effective extinction coefficients.

$$
n=\sqrt{\frac{\left|\varepsilon_{\text {eff }}\right|+\operatorname{Re}\left(\varepsilon_{\text {eff }}\right)}{2}} ; \quad k=\sqrt{\frac{\left|\varepsilon_{\text {eff }}\right|-\operatorname{Re}\left(\varepsilon_{\text {eff }}\right)}{2}}
$$

Allowing the effective refractive index, derived from (Eq.7 and Eq.11), to be considered as a thin-film enables the established metamaterial composite to be designed around the principles of a stepped index coating. The ability to accurately describe the effective thin-film in terms of an equivalent model enables an easy design route through established thin-film design techniques. 
The effective complex refractive index shown in Figure 5, consists of Silver spherical nanoparticles organized in a threedimensional primitive hexagonal Bravais lattice configuration and embedded in a high-index dielectric of Zinc Sulphide. The Zinc Sulphide material has the correct refractive index to tune the linear dependence of the localised surface plasmon resonance found in silver spherical nanoparticles at optical frequencies. Plasmons are a phenomenon where the electrons in the metals surface layer become excited by photons from the incident light resulting in the electron absorbing the photon. Silver nanoparticles were selected due to the materials flexibility in resonance engineering, particularly in the visible spectrum. Its peak wavelength, bandwidth and transmission rate can be tuned by size, surrounding medium and three-dimensional configuration. The inter particle distance between the silver nanoparticles remain fixed at $\lambda \_$laser $/ 16$. This configuration allows the resonance peak to match the laser wavelength of $532 \mathrm{~nm}$.

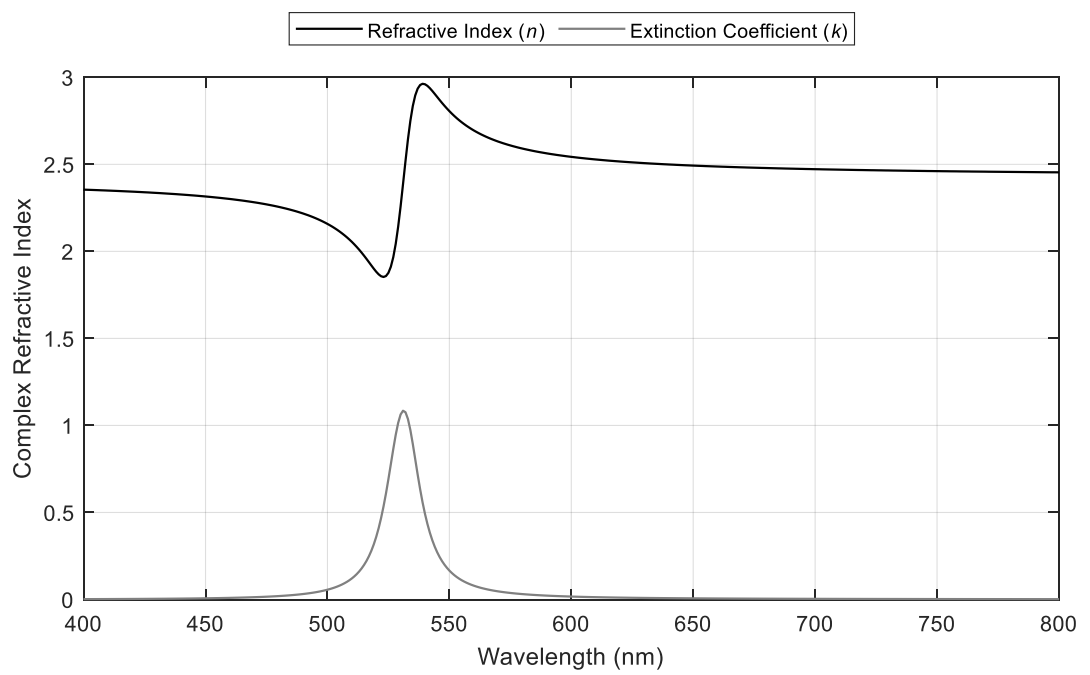

Figure 5. The effective complex refractive index of the metamaterial layer.

\section{ANGLE-INSENSITIVE METAMATERIAL FILTER}

Effects that dominate angular and polarisation insensitivity at optical frequencies rely on the metamaterials ability to create a strong electric resonance at a selected wavelength which can create circulating currents to efficiently drive a magnetic response [16]. The strong electric and magnetic responses lead to an impedance mismatch, and angular stability remains as a result of the symmetry and equivalent inductance of the meta-atom [17]. Lossy materials are the key common materials to achieve this due to the presence of the free electrons. Therefore, in order to achieve insensitivity to the incidence angle and polarisation, the metamaterial must contain an absorbing feature. The basic idea of employing the metamaterial for applications in laser protection is to design a material that induces an absorbing resonant feature at the laser wavelength.

The metamaterial design, with comparable bandwidth, attenuation, optical density, coloration, and integrated visual transmission can be found in (Eq.12). The formation of the stack is noticeably smaller when compared with the thin-film alternative. The configuration consists of four layers, a low $(L)$, high $(H)$ and medium $(M)$ index dielectric layers which combines to act as an anti-reflection coating to the metamaterial $\left(E_{M M}\right)$ layer.

$$
\text { Air }\left|\operatorname{LHM}\left(E_{M M}\right)\right| \text { Glass }
$$

The atypical, yet functionally designed curves are displayed in Figure 6 and show how the central wavelength and effective refractive index modulus of the metamaterial filter experience negligible shift around the central $532 \mathrm{~nm}$ wavelength. The modulus of the effective index is taken because unlike the thin-film equivalent, the metamaterial solution provides a complex effective index due to the absorbing properties. 


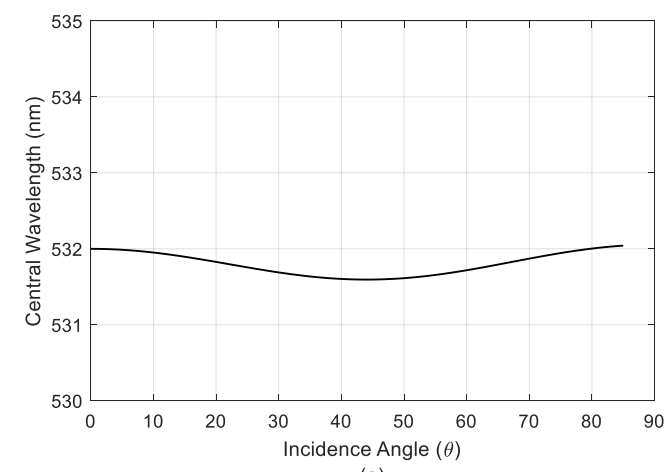

(a)

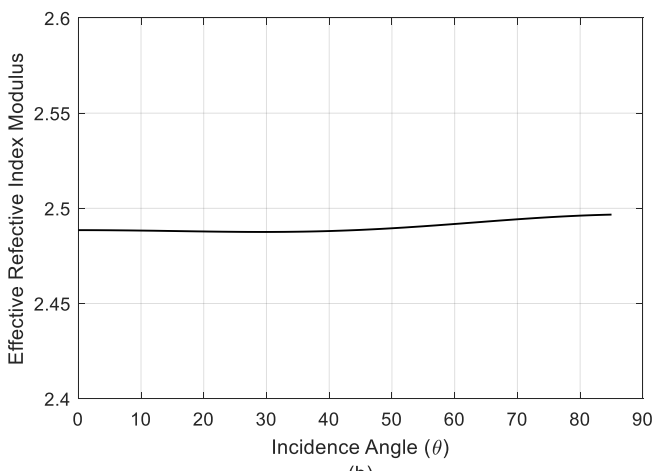

(b)

Figure 6. (a) The peak wavelength with angle of incidence. (b) The modulus effective refractive index of the stack against angle of incidence.

A design function of shift-free filters allow the polarisation, at the central wavelength, to remain unaltered and nondiverging. Outside the notch location, the polarisation naturally experiences some divergence due to the anti-reflection dielectric coating. Figure 7 shows the laser protection filter enduring a fixed bandstop location for both incidence angle and polarisation states.

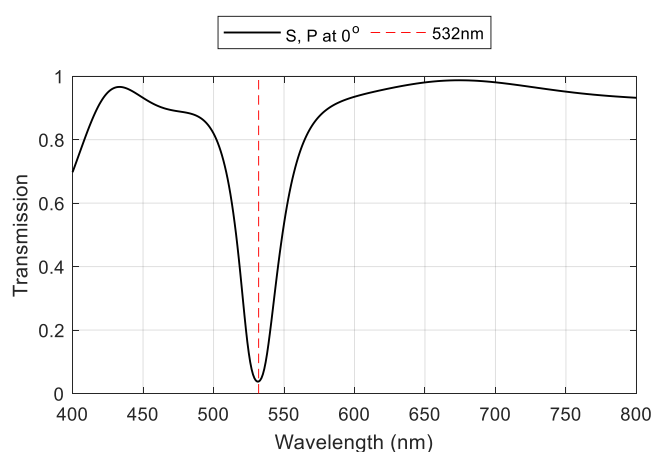

(a)

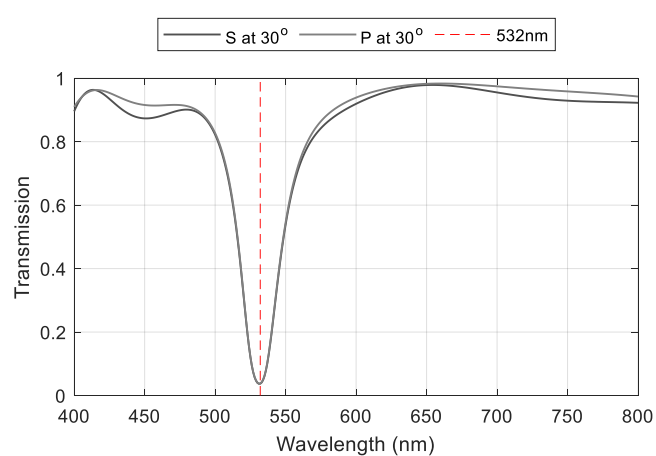

(b)

Figure 7. The transmission response with consideration to the polarisation effects on the metamaterial filter.

Figure 8 , highlights the mean transmission function of the shift-free metamaterial filter at incidence angle 0,25 and 50 degrees. The dotted line represents the laser wavelength. The figure focuses on the bandstop region to demonstrate how the notch location remains fixed to the resonant wavelength and has the ability to provide adequate protection, without loss of attenuation, against $532 \mathrm{~nm}$ laser wavelength. As the incidence angle increases, the notch becomes asymmetric but does not deviate from the desired blocking wavelength. 


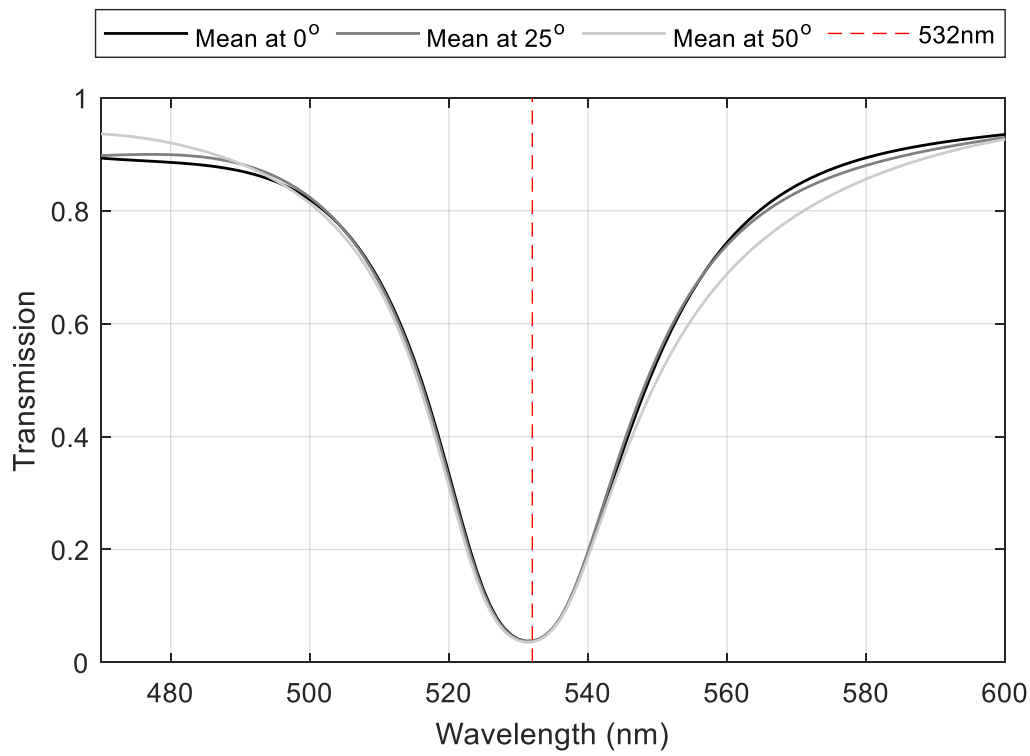

Figure 8. The mean transmission function of the metamaterial filter, focusing on the bandstop region.

As previously mentioned, the optical density is commonly used to define laser protection filters. Figure 9 shows the calculated optical density of the metamaterial filter for angles between 0 and 85 degrees at a fixed wavelength of 532 $\mathrm{nm}$. Unlike the thin-film filter that tends to zero OD above 30 degrees, the metamaterial filter remains static just below $1.5 \mathrm{OD}$ to 50 degrees. At 50+ degrees, the OD begins to increase at a non-linear rate. Contrasting to the decrease in the thin-film filter, which occurs due to the notch shifting to smaller wavelength; the increase in optical density for the metamaterial filter is predominately due to an increased attenuation at larger incidence angles. The optical density plot in Figure 9, clearly demonstrates the metamaterial filter's capability to provide protection at $532 \mathrm{~nm}$ across all angles of incidence. High optical density can be achieved by increasing the metamaterial layer thickness, but at the expensive of increasing the bandwidth.

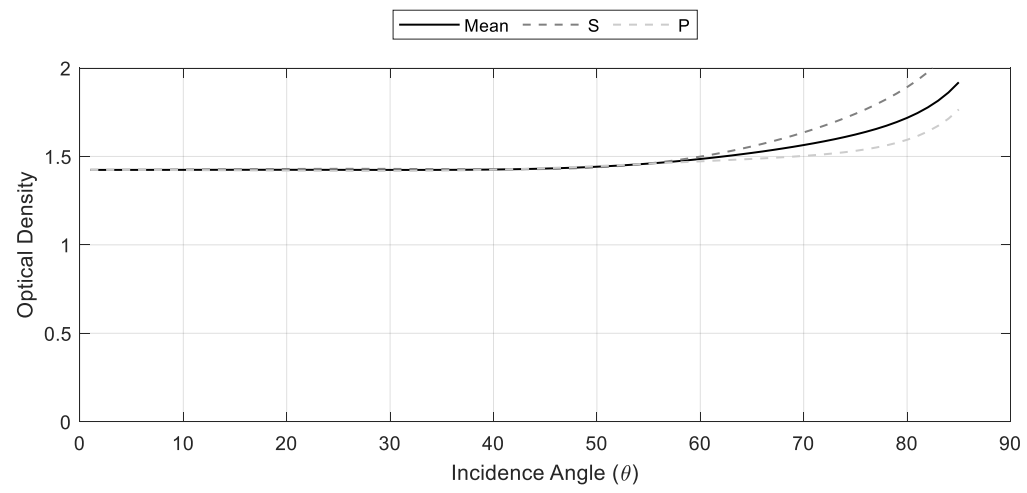

Figure 9. Optical density as a function of incidence angle at a fixed wavelength of $532 \mathrm{~nm}$. 


\section{CONCLUSION}

Standard thin-film interference filters have provided protection against lasers since the late 1970s. Nonetheless, with the growing demand in laser protection technologies, thin-film filters are lacking the fundamental safeguarding against incidence laser light from varying angles due to the shifting nature. With the growing trends of metamaterials and the ability to design functionality directly into a material, this new technology could present the solution to this problem. This research has highlighted important shortcomings in thin-films filters, for application in laser protection and introduced a metamaterial alternative with routes for designing such materials. The design discussed offers a single notch filter to protect against a $532 \mathrm{~nm}$ laser. The effective Drude-Lorentz approximation model for dispersive metamaterials offers a simpler alternative to the Maxwell-Garnett solution with metallic inclusions. The metamaterial design principles put forward in this research could be used to develop alternative bandstop filters to protect against other laser wavelengths. The process and outcome of this research is pushing the foundations of fixed-line laser protection filter technology to new, unimaginable limits that exceeds conventional dielectric interference filters, and aims for more efficient laser countermeasures.

\section{REFERENCES}

[1] Kalyani, V., Sharma, V., "Different types of Optical Filters and their Realistic Application", Journal of Management Engineering and Information Technology 3(3), (2016).

[2] Macleod, H., Thin-film optical filters, 3rd ed., Institute of Physics Publishing, Philiadelphia (2001).

[3] Baker, M., Yen, V., "Effects of the Variation of Angle of Incidence and Temperature on Infrared Filter Characteristics", Applied Optics 6(8), 1343 (1967).

[4] Monks, J., Yue, L., Yan, B., Aldred, B., Hurst, A., Wang, Z., "A wide-angle shift-free metamaterial filter design for anti-laser striking application", Optics Communications 429, 53-59 (2018).

[5] Liu, Y., Zhang, X., "Metamaterials: a new frontier of science and technology", Chemical Society Reviews 40(5), 2494 (2011).

[6] Corbitt, S., Francoeur, M., Raeymaekers, B., "Implementation of optical dielectric metamaterials: A review", Journal of Quantitative Spectroscopy and Radiative Transfer 158, 3-16 (2015).

[7] Urbas, A., Jacob, Z., Negro, L., Engheta, N., Boardman, A., Egan, P., Khanikaev, A., Menon, V., Ferrera, M. et al., "Roadmap on optical metamaterials", Journal of Optics 18(9), 093005 (2016).

[8] Markel, V., "Maxwell Garnett approximation (advanced topics): tutorial", Journal of the Optical Society of America A 33(11), 2237 (2016).

[9] Zhang, X., Wu, Y., "Effective medium theory for anisotropic metamaterials", Scientific Reports 5(1) (2015).

[10] Pidgeon, C., Smith, S., "Resolving Power of Multilayer Filters in Nonparallel Light", Journal of the Optical Society of America 54(12), 1459 (1964).

[11] Yu, H., Peng, Y., Yang, Y., Li, Z., "Plasmon-enhanced light-matter interactions and applications", npj Computational Materials 5(1) (2019).

[12] Cai, W., Šalaev, V., Optical Metamaterials, Springer-Verlag New York, New York, NY (2010).

[13] Papadakis, G., Fleischman, D., Davoyan, A., Yeh, P., Atwater, H., "Optical magnetism in planar metamaterial heterostructures", Nature Communications 9(1) (2018).

[14] Slovick, B., Yu, Z., Krishnamurthy, S., "Generalized effective-medium theory for metamaterials", Physical Review B 89(15) (2014).

[15] Zhuromskyy, O., "Applicability of Effective Medium Approximations to Modelling of Mesocrystal Optical Properties", Crystals 7(1), 1 (2016).

[16] Zhu, W., Zhao, X., Gong, B., Liu, L., Su, B., "Optical metamaterial absorber based on leaf-shaped cells", Applied Physics A 102(1), 147-151 (2010).

[17] Bagci, F., Akaoglu, B., "Consequences of Unit Cell Design in Metamaterial Perfect Absorbers", Acta Physica Polonica A 129(4), 792-796 (2016). 